\title{
Aerosol Delivery Enhances Percutaneous Permeability of Epidermal Growth Factor and Thioredoxin
}

Keywords: Aerosol delivery; Thioredoxin; Epidermal growth factor; Percutaneous permeability

\begin{abstract}
To evaluate the effect of a nano-spray instrument, we examined the transcutaneous permeability of human recombinant thioredoxin (rhTRX) and human recombinant epidermal growth factor (rhEGF). Distribution of rhTRX and rhEGF in skin tissues was studied by immunohistochemical analyses after their application. At least $1 \mathrm{\mu g} / \mathrm{ml}$ rhEGF was distributed in the epidermis after application using the nano-spray instrument. Permeability of rhEGF decreased if the spraying time was shorter or the spraying distance was longer. Sprayed rhTRX was distributed more densely in the epidermis, and could strongly suppressed irritant contact dermatitis induced by croton oil, compared with the painted rhTRX group. These data suggest that aerosol delivery is a safe and effective method of enhancement of drug permeation into skin, and could be used to improve the effects of drugs.
\end{abstract}

\section{Introduction}

To attain the desired effect of a medication by topical application, the medication must become permeabilized in skin. However, skin is an exceptionally effective barrier to prevent permeation of drugs applied for therapy [1,2]. Few drugs can penetrate in significant amounts into skin. Most of the topical dosage forms available commercially have poor penetration, which can lead to poor therapeutic benefits $[3,4]$. Therefore, development of a method of topical application that makes the skin more permeable and that enables skin penetration by multiple mechanisms to enhance delivery of topical drugs is important.

Two strategies to improve drug permeation of skin have been attempted: chemical and physical. Chemical approaches are enhancers such as alcohol, monoterpenes, and fatty acid esters, as well as chemical modification of prodrugs. Physical approaches include use of energy (electrical, thermal, mechanical), as well as micro needles, needle-free injectors, or electroporation to completely or partially evade the barrier function in the stratum corneum.

Human epidermal growth factor (EGF) is a 6-kDa polypeptide of 53 amino acids that promotes cell growth by binding to its receptor on the cell surface [5]. EGF is an important autocrine/paracrine factor in the human body. EGF is used in pharmaceutical/cosmetic industries to prevent post-inflammatory hyperpigmentation, presumably by accelerating the turnover or wound healing of skin.

Human thioredoxin (TRX) is a $12-\mathrm{kDa}$ protein with redoxactive dithiol in the active site (-Cys-Gly-Pro-Cys-). It is induced by oxidative-stress factors such as ultraviolet rays, radiation, oxidation,

\section{Journal of}

\section{Clinical \& Investigative} Dermatology

\author{
Hai Tian' ${ }^{1}$ Atsushi Fukunaga ${ }^{2}$, Junji Yodoi ${ }^{3}$ and Chikako \\ Nishigori ${ }^{2}$ \\ ${ }^{\prime}$ Redox Bio Science Incorporated, Kyoto, Japan \\ ${ }^{2}$ Division of Dermatology, Department of Internal Related, Kobe University \\ Graduate School of Medicine, Kobe, Japan \\ ${ }^{3}$ Department of Biological Response, Institute for Virus Research, Kyoto \\ University, Kyoto, Japan \\ *Address for Correspondence \\ Atsushi Fukunaga, Division of Dematology, Department of Intemal Related, Kobe \\ University Graduate School of Medicine, 7-5-1 Kusunoki-cho Chuo-ku Kobe, 650-0017, \\ Japan, Tel: 81-78-382-6138; Fax: 81-78-382-6149; E-mail: atsushi@med.kobe-u.ac.jp \\ Submission: 01 December, 2015 \\ Accepted: 23 January, 2016 \\ Published: 29 January, 2016 \\ Copyright: ๑ 2016 Tian H, et al. This is an open access article distributed \\ under the Creative Commons Attribution License, which permits unrestricted \\ use, distribution, and reproduction in any medium, provided the original work \\ is properly cited.
}

viral infections, ischemia-reperfusion, and anticancer agents [6]. We have demonstrated that exogenous TRX is effective in several models of inflammatory disease in vivo: viral pneumonia, acute lung injury, pancreatitis, myocarditis, chronic obstructive pulmonary disease, indomethacin-induced gastric injury, and dermatitis [7-13].

Our recent study suggested that recombinant human TRX (rhTRX) administered exogenously can suppress irritant contact dermatitis (ICD) by inhibition of local formation of inflammatory cytokines and chemokines [14]. That study also suggested that TRX applied externally could be useful for the treatment of skin disorders and mucosal disorders [14].

In the present study, to evaluate the effect of aerosol delivery for percutaneous permeability of proteins, recombinant human EGF (rhEGF) and rhTRX were applied topically by a nano-spray instrument (NSI) on the surface of mouse skin, and the skin permeability of rhEGF and rhTRX investigated. In addition, the antiinflammatory effect of rhTRX sprayed by a NSI was compared with that of painted rhTRX in the well-established model of ICD induced by croton oil $[13,14]$.

\section{Materials and Methods}

\section{Nano-spray instrument (NSI)}

NSI uses the ultrasonic vibration and atomization technology to transform skin care lotion into nano-scale spray so as to enhance penetration to the skin for better absorption. The Nano spray with ultra-high frequency of $2.4 \mathrm{MHz}$ can make the effective component to tiny Nano-meter particles for deep permeating. The NSI was gained form Konishi Seiko Co., Ltd., Kyoto, Japan as a gift (Figure 1).

\section{Mice}

Wild-type female C57BL6 mice (8 weeks) were purchased from Charles River Japan (Tokyo, Japan). All animals were maintained in micro-isolator cages. Mice were exposed to a $12 \mathrm{~h}$ light-dark cycle with 
Citation: Tian H, Fukunaga A, Yodoi J, Nishigori C. Aerosol Delivery Enhances Percutaneous Permeability of Epidermal Growth Factor and Thioredoxin. J Clin Investigat Dermatol. 2016;4(1): 5.

free access to standard mice chow and water. All experiments were conducted according to the Institutional guidelines and regulations.

\section{Topical application of rhEGF}

rhEGF (1-30 $\mu \mathrm{g} / \mathrm{ml}$; R\&D Systems, Minneapolis, MN, USA) in phosphate-buffered saline (PBS) was sprayed topically using a NSI (Konishi Seiko, Kyoto, Japan) (Figure 1) on the dorsal and ventral aspects of the ears (time, 10,20,30 s; distance, $5,10,15 \mathrm{~cm}$, respectively). Only PBS was applied as a negative control.

\section{Topical application of rhTRX}

rhTRX $(20 \mu \mathrm{g} / \mathrm{ml}$; Redox Bio Science, Kyoto, Japan) in PBS was sprayed topically by a NSI (Konishi Seiko) (Figure 1) on the dorsal and ventral aspects of the ears (time, $5 \mathrm{~s}$; distance, $10 \mathrm{~cm}$ ), and $20 \mu \mathrm{g} /$ $\mathrm{ml}$ of bovine serum albumin (BSA) applied as a control. rhTRX (20 $\mu \mathrm{g} / \mathrm{ml})$ in $2 \%$ hydrogel was painted on the dorsal aspects of the ears, and BSA $(20 \mu \mathrm{g} / \mathrm{ml})$ in $2 \%$ hydrogel was applied as a control.

\section{ICD model}

Ten microliters of $2 \%$ croton oil (Crotonis oleum) in acetone/ olive oil (Sigma-Aldrich, Saint Louis, MO, USA) was applied to the dorsal and ventral aspects of both sides of the ears. Mice were divided into four groups depending on what was applied immediately after treatment with croton oil: (i) painted with rhTRX in $2 \%$ hydrogel ( $\mathrm{n}=6)$; (ii) painted with BSA in $2 \%$ hydrogel $(\mathrm{n}=6)$; (iii) sprayed with $\operatorname{rhTRX}(\mathrm{n}=5)$; (iv) sprayed with BSA $(\mathrm{n}=5)$. Ear swelling was measured in a blinded fashion with a Digimatic Micrometer (Mitutoyo, Tokyo, Japan) $6 \mathrm{~h}$ or $24 \mathrm{~h}$ after treatment with croton oil.

\section{Immunohistochemical (IHC) analyses}

Briefly, tissue samples were prepared as sections (thickness, $3 \mu \mathrm{m})$ from paraffin-embedded specimens. This was followed by deparaffinization and blocking of endogenous peroxidase activity with $3 \%$ hydrogen peroxide in methanol for $15 \mathrm{~min}$. Then, $10 \%$

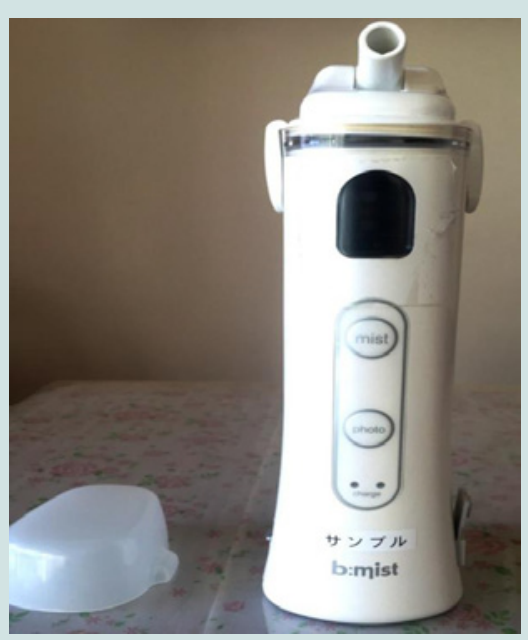

Figure 1: The nano-spray instrument is nanometer atomization facia beautification instrument, which is adopted with the ultrasonic vibration and atomization technology. It can atomize the water/lotion (without oil) into ultrafine particles with diameter of $3-5 \mu \mathrm{m}$ by vibrating millions times per second. The velocity of the particles is almost $4.1-4.2 \mathrm{~m} / \mathrm{s}$ when they are expelled from the nozzle exit. The flow rate of the mist was $1 \mathrm{ml} / \mathrm{min}$.

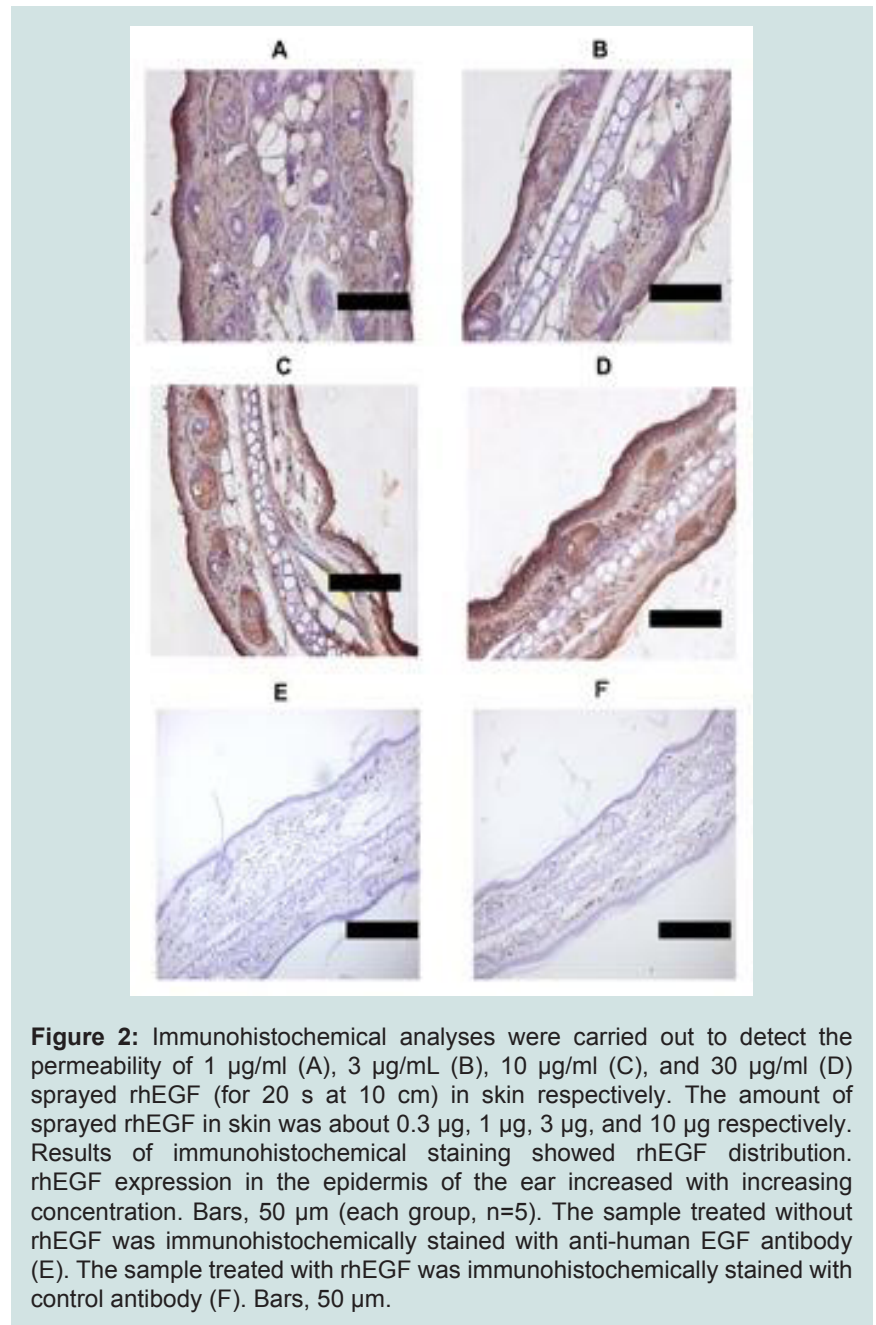

BSA was added for 30 min to block non-specific binding at room temperature. Tissue specimens were incubated with a mouse antihuman EGF monoclonal antibody (R\&D Systems) and a mouse antihuman monoclonal TRX antibody (Redox Bio Science) overnight at 4 ${ }^{\circ} \mathrm{C}$. After washing with PBS, sections were treated at room temperature with a Histofine Mouse Stain kit (Nichirei Bioscience, Tokyo, Japan) to block non-specific binding, then with 3,3-diaminobenzidine working solution (Vector Laboratories, Burlingame CA, USA) or 3-amino-9-ethylcarbazole substrate (Nichirei Bioscience). Tissue sections were counterstained with hematoxylin.

\section{Statistical analyses}

Results are the mean $\pm \mathrm{SD}$. The Student's $t$-test was used for comparisons between two groups. $\mathrm{p}<0.05$ was considered significant.

\section{Results}

\section{Sprayed rhEGF was distributed in the epidermis}

First, rhEGF of low molecular weight was applied to skin to examine the effect of the NSI. To investigate the optimal concentration of rhEGF, $1-30 \mu \mathrm{g} / \mathrm{ml} \mathrm{rhEGF}$ was sprayed onto skin $(20 \mathrm{~s}$ at $10 \mathrm{~cm}$ ), and rhEGF permeability in skin investigated $24 \mathrm{~h}$ later by IHC analyses. Sprayed rhEGF was distributed mainly in the epidermis 
Citation: Tian H, Fukunaga A, Yodoi J, Nishigori C. Aerosol Delivery Enhances Percutaneous Permeability of Epidermal Growth Factor and Thioredoxin. J Clin Investigat Dermatol. 2016;4(1): 5.

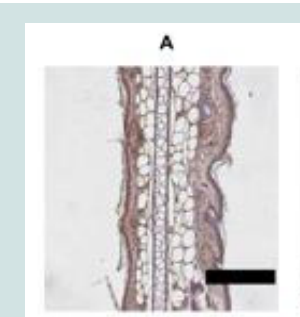

D

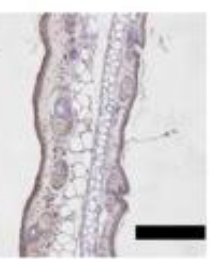

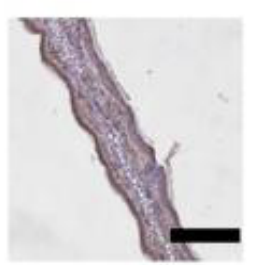
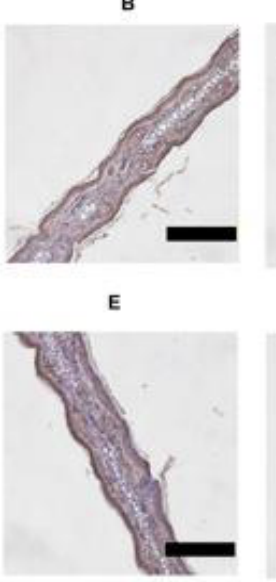

c

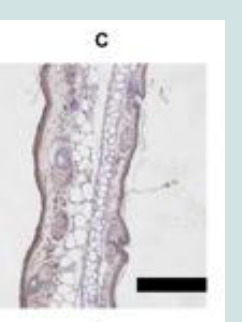

$\mathbf{F}$

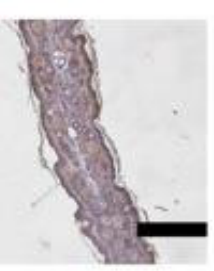

Figure 3: Immunohistochemical analyses showed that rhEGF permeability changed with variation in spraying time and distance $A$. (10 s at $5 \mathrm{~cm}), B$. (10 $\mathrm{s}$ at $10 \mathrm{~cm}), C(10 \mathrm{~s}$ at $15 \mathrm{~cm}), D .(10 \mathrm{~s}$ at $15 \mathrm{~cm}), E .(20 \mathrm{~s}$ at $15 \mathrm{~cm})$, and F. (30 $\mathrm{s}$ at $15 \mathrm{~cm}$ ). Sprayed rhEGF permeability increased as spraying time increased or spraying distance decreased. (Bars, $50 \mu \mathrm{m}$ (each group, $\mathrm{n}=5$ ).

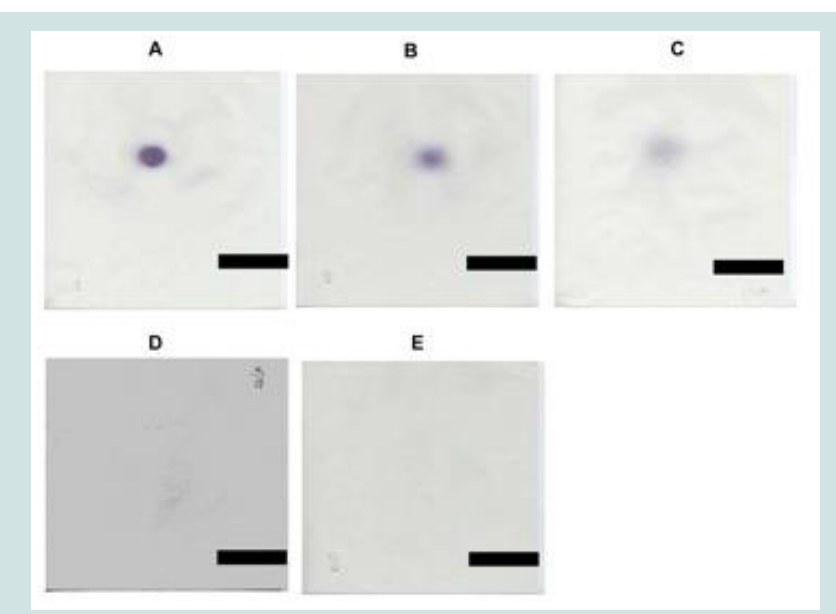

Figure 4: Spraying area of $50 \%$ hematoxylin was dependent upon the change in spraying distance A. $(5 \mathrm{~cm})$, B. $(10 \mathrm{~cm})$, C. $(15 \mathrm{~cm}), D .(20 \mathrm{~cm})$, and E. $(25 \mathrm{~cm})$. When the spraying distance was longer, hematoxylin deposition became weaker, and spraying area increased. The diameters of hematoxylin depositions were $2.5 \mathrm{~cm}$ (A) $3.5 \mathrm{~cm}$ (B) $4.5 \mathrm{~cm}$ (C) and $6 \mathrm{~cm}$ (D) respectively, but the deposition was not found when the spray distance was $25 \mathrm{~cm}$.

and cutaneous appendages, and rhEGF expression increased with increasing concentration (Figure 2). rhEGF was detected in skin using $3 \mu \mathrm{g} / \mathrm{ml} \mathrm{rhEGF}$, therefore this concentration was selected for subsequent experiments. Then, to examine the optimal spraying time and optimal distance, $3 \mu \mathrm{g} / \mathrm{ml} \mathrm{rhEGF}$ was applied for 10,20 and 30 $\mathrm{s}$, and 5,10 and $15 \mathrm{~cm}$ from the NSI to the skin, respectively. rhEGF permeability increased as the spraying time increased or spraying distance decreased (Figure 3). These findings suggested that rhEGF penetrated the epidermis using the NSI. Last, to evaluate the area of mist created, $50 \%$ hematoxylin in distilled water was sprayed 5-25 $\mathrm{cm}$ from the NSI to the paper for $10 \mathrm{~s}$ instead of rhEGF. Then, the diameters of hematoxylin depositions were measured by the ruler. Hematoxylin deposition decreased and the area of mist expanded as the spray distance increased (Figure 4).

\section{Sprayed rhTRX penetrated more strongly into the epidermis than painted rhTRX}

Next, to further examine the permeability of the NSI compared with simple application, rhTRX of higher molecular weight was applied to skin. rhTRX was distributed mainly on the horny layer and the surface of the epidermis $6 \mathrm{~h}$ after simple application (painting), whereas sprayed rhTRX penetrated into the entire epidermis $6 \mathrm{~h}$ later (Figure 5). Anti-human TRX antibodies did not cross-react with mouse TRX [14], confirming that rhTRX applied only exogenously was detected upon histological analyses. These findings suggested that larger protein molecules and small protein molecules could penetrate into the epidermis using a NSI.

\section{Sprayed rhTRX suppressed ICD more strongly than painted rhTRX}

TRX has excellent anti-inflammatory effects for treatment of various inflammatory diseases in various animal models [7-14]. Hence, we undertook a comparative study using a model of ICD on the anti-inflammatory effects of sprayed rhTRX and painted rhTRX. Sprayed rhTRX and painted rhTRX significantly suppressed ear swelling $6 \mathrm{~h}$ after application of croton oil, whereas the inhibitory effect of ear swelling on sprayed rhTRX tend to be stronger than on painted rhTRX $(\mathrm{p}<0.001 v s \mathrm{p}<0.05$; spray vs paint). Sprayed rhTRX also suppressed ear swelling significantly $24 \mathrm{~h}$ after treatment with croton oil $(\mathrm{p}<0.001)$, but not painted rhTRX (Figure 6A). Though the significant inflammatory response of skin such as edema, neutrophil infiltration was strongly suppressed in both rhTRX sprayed group and rhTRX painted group (Figures 6B-6E). These data suggested that transcutaneous application of rhTRX by a NSI has an equivalent effect

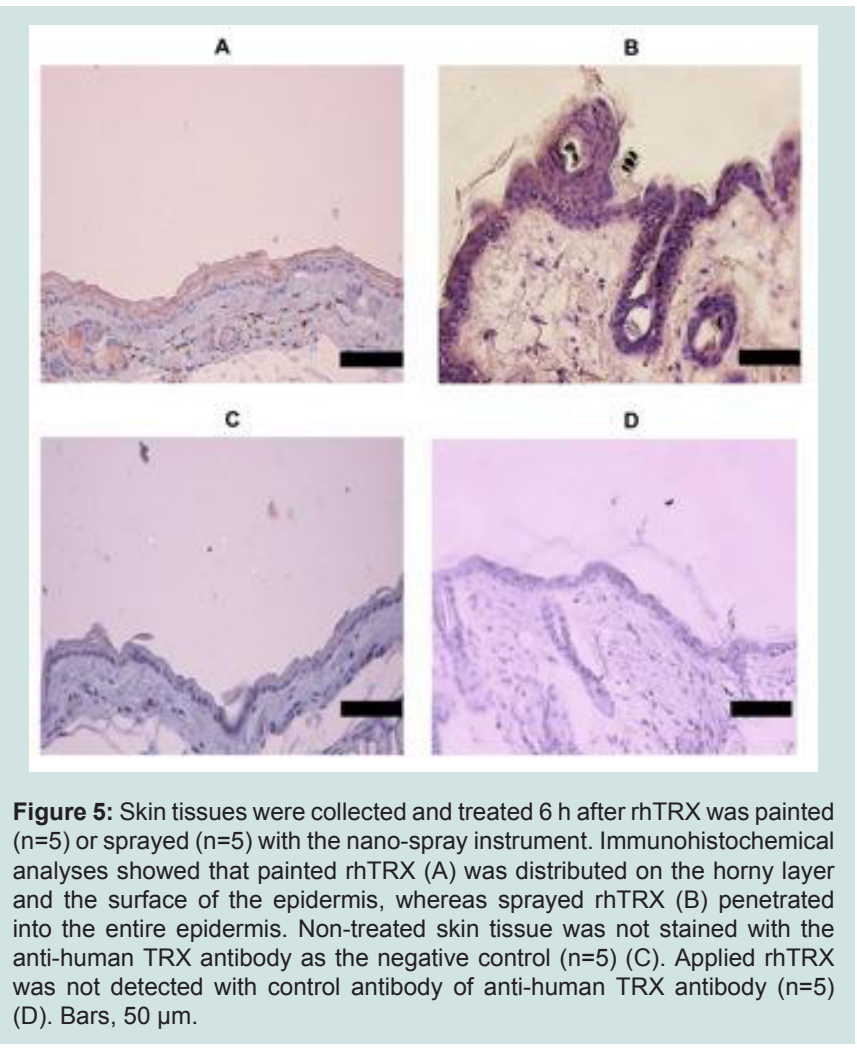


Citation: Tian H, Fukunaga A, Yodoi J, Nishigori C. Aerosol Delivery Enhances Percutaneous Permeability of Epidermal Growth Factor and Thioredoxin. J Clin Investigat Dermatol. 2016;4(1): 5.

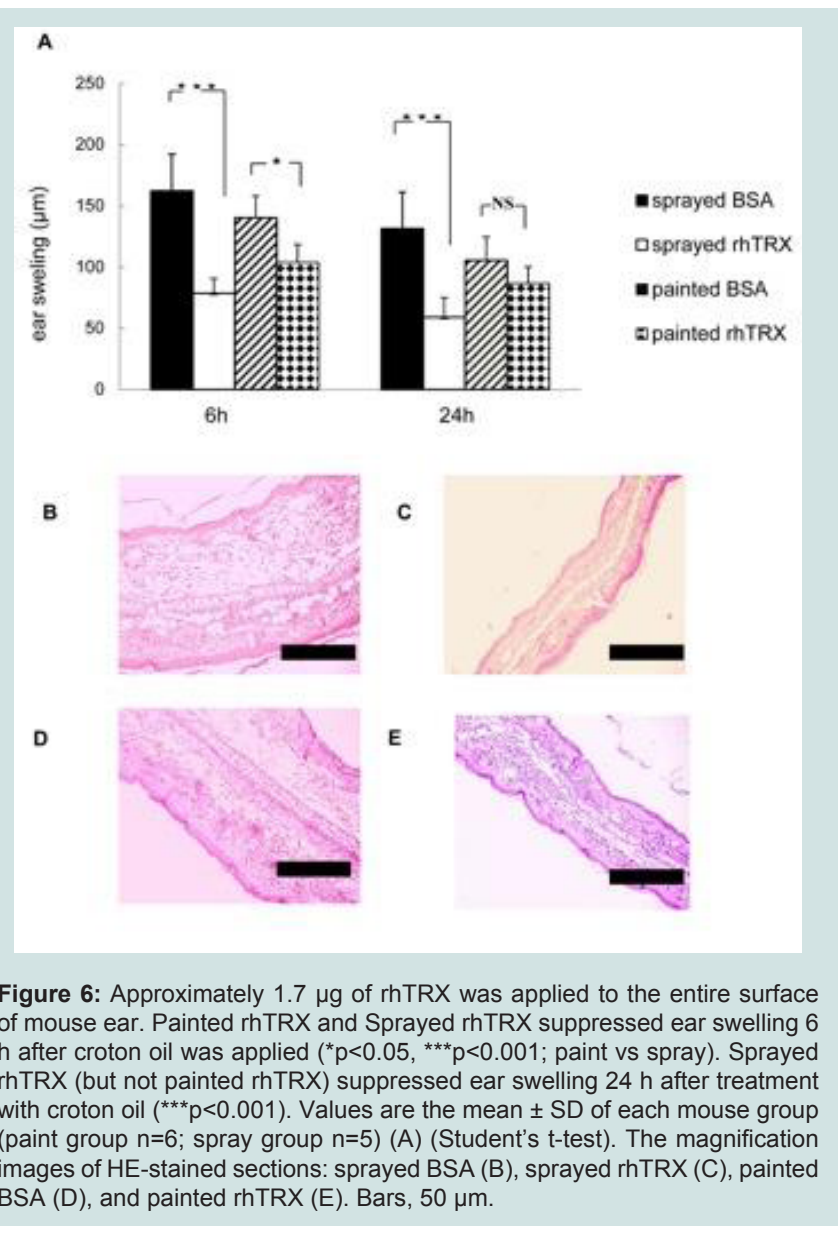

with a simple application and/or might enhance anti-inflammatory effects on skin.

\section{Discussion}

The barrier of skin disturbs permeation of medicines and cosmetics into the dermis as well as defending against antigens and toxic substances $[15,16]$. Several penetration-enhancing approaches have been developed (e.g., chemicals, skin-ablation devices) [17]. However, the cost and inconvenience of these strategies highlights the need for a novel and safe method for increasing skin absorption.

In the present study, a NSI that could change conventional mist into ultrafine particles was used to improve transcutaneous permeation of proteins. We demonstrated that a NSI could improve the permeability of rhEGF and rhTRX into skin under an optimal condition, suggesting that the NSI was an enhancer of transcutaneous permeability. rhTRX is a water-soluble agent and its application to skin was difficult, but rhTRX application using the NSI was relatively easy. In addition, the NSI could spray over a relatively wide range if the spraying distance was longer. These data suggested that drug delivery of aerosols using the NSI was practical and confirmed the improvement of transcutaneous permeability. In addition, aerosol delivery of rhTRX using the NSI enhanced the anti-inflammatory effects against ICD.

EGF has been used for several types of cosmetics, and the role of EGF in wound healing has been examined in human and animal models $[18,19]$. In addition, topical application of EGF accelerates dermal regeneration of partial thickness or second-degree burn wounds [20,21]. However, most products containing EGF are applied topically to wounds [22]. Efficacy of EGF products is limited by its poor transdermal permeability and biological stability [23]. We investigated the optimal spraying condition of rhEGF. Even $1 \mu \mathrm{g} / \mathrm{ml}$ EGF penetrated into the epidermis after it was sprayed for $20 \mathrm{~s}$ at 10 $\mathrm{cm}$ by the NSI. This finding suggests that aerosol delivery can be used as delivery methods of EGF products.

Previously, we demonstrated that rhTRX administered exogenously can suppress allergic contact dermatitis and ICD $[13,14]$. In the present study, aerosol delivery of rhTRX using the NSI enhanced the anti-inflammatory effects against ICD compared with simple application of rhTRX. Moreover, the anti-inflammatory effects with aerosol delivery of rhTRX lasted for $24 \mathrm{~h}$. Collectively, these data suggest that aerosol-based transcutaneous delivery of anti-inflammatory proteins may be more useful for the treatment of dermatitis than simple application.

\section{Conclusion}

We demonstrated that the permeability of proteins into skin was enhanced by a NSI. Aerosol delivery of proteins as skin-permeation enhancers could be applied for cosmetic treatments as well as for treatments of inflammatory diseases in the skin or mucosa.

\section{References}

1. Lee SE, Choi Y, Kim SE, Noh EB, Kim SC (2013) Differential effects of topical corticosteroid and calcineurin inhibitor on the epidermal tight junction. Exp Dermatol 22: 59-61.

2. Marto J, Baltazar D, Duarte A, Fernandes A, Gouveia L, et al. (2015) Topical gels of etofenamate: in vitro and in vivo evaluation. Pharm Dev Technol 20: 710-715.

3. Lau WM, White AW, Gallagher SJ, Donaldson M, McNaughton G, et al (2008) Scope and limitations of the co-drug approach to topical drug delivery. Curr Pharm Des 14: 794-802.

4. Raza K, Kumar M, Kumar P, Malik R, Sharma G, et al. (2014) Topical delivery of aceclofenac: challenges and promises of novel drug delivery systems. Biomed Res Int 2014: 406731.

5. Carpenter G, Cohen S (1990) Epidermal growth factor. J Biol Chem 265: 7709-7712.

6. Holmgren A (1985) Thioredoxin. Annu Rev Biochem 54: 237-271.

7. Nakamura H, Tamura Si, Watanabe I, Iwasaki T, Yodoi J (2002) Enhanced resistancy of thioredoxin-transgenic mice against influenza virus-induced pneumonia. Immunol Lett 82: 165-170.

8. Ueda S, Nakamura T, Yamada A, Teratani A, Matsui N, et al. (2006) Recombinant human thioredoxin suppresses lipopolysaccharide-induced bronchoalveolar neutrophil infiltration in rat. Life Sci 79: 1170-1177.

9. Ohashi S, Nishio A, Nakamura H, Kido M, Ueno S, et al. (2006) Protective roles of redox-active protein thioredoxin-1 for severe acute pancreatitis. Am J Physiol Gastrointest Liver Physiol 290: G772-G781.

10. Liu W, Nakamura H, Shioji K, Tanito M, Oka S, et al. (2004) Thioredoxin-1 ameliorates myosin-induced autoimmune myocarditis by suppressing chemokine expressions and leukocyte chemotaxis in mice. Circulation 110: 1276-1283.

11. Hoshino Y, Nakamura T, Sato A, Mishima M, Yodoi J, et al. (2007) Neurotropin demonstrates cytoprotective effects in lung cells through the induction of thioredoxin-1. Am J Respir Cell Mol Biol 37: 438-446. 
Citation: Tian H, Fukunaga A, Yodoi J, Nishigori C. Aerosol Delivery Enhances Percutaneous Permeability of Epidermal Growth Factor and Thioredoxin. J Clin Investigat Dermatol. 2016;4(1): 5.

12. Nakajima A, Fukui T, Takahashi Y, Kishimoto M, Yamashina M, et al. (2012) Attenuation of indomethacin-induced gastric mucosal injury by prophylactic administration of sake yeast-derived thioredoxin. J Gastroenterol 47: 978987.

13. Fukunaga A, Horikawa T, Ogura K, Taguchi K, Yu X, et al. (2009) Thioredoxin suppresses the contact hypersensitivity response by inhibiting leukocyte recruitment during the elicitation phase. Antioxid Redox Signal 11: 12271235 .

14. Tian H, Matsuo Y, Fukunaga A, Ono R, Nishigori C, et al. (2013) Thioredoxin ameliorates cutaneous inflammation by regulating the epithelial production and release of pro-inflammatory cytokines. Front Immunol 4: 269

15. Andrews SN, Jeong E, Prausnitz MR (2013) Transdermal delivery of molecules is limited by full epidermis, not just stratum corneum. Pharm Res 30: 1099-1109.

16. Barry BW (2004) Breaching the skin's barrier to drugs. Nat Biotechnol 22 165-167.

17. Akomeah F, Nazir T, Martin GP, Brown MB (2004) Effect of heat on the percutaneous absorption and skin retention of three model penetrants. Eur J Pharm Sci 21: 337-345.
18. Franklin JD, Lynch JB (1979) Effects of topical applications of epidermal growth factor on wound healing. Experimental study on rabbit ears. Plast Reconstr Surg 64: 766-770.

19. Niall M, Ryan GB, O'Brien BM (1982) The effect of epidermal growth factor on wound healing in mice. J Surg Res 33: 164-169.

20. Thornton JW, Hess CA, Cassingham V, Bartlett RH (1982) Epidermal growth factor in the healing of second degree burns: a controlled animal study. Burns Incl Therm Inj 8: 156-160.

21. Arturson G (1984) Epidermal growth factor in the healing of corneal wounds, epidermal wounds and partial-thickness scalds. A controlled animal study. Scand J Plast Reconstr Surg 18: 33-37.

22. Lee JH, Bae IH, Choi JK, Park JW (2013) Evaluation of a highly skin permeable low-molecular-weight protamine conjugated epidermal growth factor for novel burn wound healing therapy. J Pharm Sci 102: 4109-4120.

23. Choi JK, Jang JH, Jang WH, Kim J, Bae IH, et al. (2012) The effect of epidermal growth factor (EGF) conjugated with low-molecular-weigh protamine (LMWP) on wound healing of the skin. Biomaterials 33: 8579-8590.

\section{Acknowledgements}

We greatly appreciate the technical advice of Dr. Yoshiyuki Matsuo (Laboratory of Infection and Prevention, Department of Biological Response, Institute for Virus Research, Kyoto University, Kyoto, Japan). This study was supported by the World Class University Grant R31-10010 through the Ewha Womans University (Seoul, South Korea). 